\title{
Option pricing in the binomial model
}

Jasmine Vestman

Examensarbete i matematik, $15 \mathrm{hp}$ Handledare: Erik Ekström Examinator: Martin Herschend Juni 2021 



\begin{abstract}
In this thesis, we will cover basic option theory and its pricing in the binomial model. We will first look at the one-period model and then move on to the multiperiod model. Furthermore, I will look at the choice of the volatility parameter in the multiperiod model. We will see that when $\Delta t \rightarrow 0$, the best choice is for it to be as close to the underlying parameter as possible. Moreover, the monotone relationship in the option price with respect to the risk-free rate will also be explored, where the results will vary depending on which model we are looking at.
\end{abstract}




\section{Contents}

1 Background 2

1.1 Stock Options . . . . . . . . . . . . . . . . . . 2

2 Binomial model 3

2.1 One-period time model . . . . . . . . . . . . . . . . . 3

2.2 Multiperiod time model . . . . . . . . . . . . . . 7

3 Volatility 11

3.1 Exploring the choice of $\sigma \ldots \ldots \ldots \ldots 11$

4 Option prices and monotonicity in rate 13

4.1 Monotonicity in the one-period model . . . . . . . . . . . . . 13

4.2 Monotonicity in the multiperiod model . . . . . . . . . . . . 14

$\begin{array}{ll}\text { References } & 16\end{array}$ 


\section{Background}

\section{$1.1 \quad$ Stock Options}

Stock options are one type of financial derivative which works as a contract between the seller and the buyer of the option. The agreement gives the option holder the right but not obligation to buy or sell stock shares at a given period in time, for a predetermined price. The predetermined price is called the strike price, and the end of the period is called the expiration date. If the option holder decides to use the option, we say that the holder is exercising the option. If the option holder is not exercising the option before or on the expiration date, it is usually because the option is out of the money. The option can be either in the money, out of the money or at the money. If the option is out of the money, the option holder will not make a financial gain from exercising the option contrary to when the option is in the money. When the option is at the money, the strike price is the same as the underlying asset. There are several types of options, and below are given examples of common options on the market today:

- European options: Gives the option holder the right but not the obligation to exercise the option at the expiration date.

- American options: Gives the option holder the right but not the obligation to exercise the option at any time up to the expiration date.

- Call options: Gives the option holder the right but not the obligation to buy a stock share for the strike price.

- Put options: Gives the option holder the right but not the obligation to sell a stock share for the strike price.

In the thesis, the primary focus will be European call and put options. Furthermore, any reference or use of the word 'price' will refer to the option's premium. The introduction of this thesis will follow Tomas Björk's book, Arbitrage Theory in Continuous Time, chapter 2. 


\section{Binomial model}

We start by defining the one-period time model, later on we will also define the multiperiod model.

\subsection{One-period time model}

Let our discrete-time model contain two financial assets, bonds and stock shares. Moreover, let $t \in\{0,1\}$ denote the running-time, $R$ the interest rate and $B_{t}, S_{t}$ denote the price of a bond and price of one share of stock at time $t$ respectively. The price process of the bond is deterministic and given by:

$$
\begin{aligned}
& B_{0}=1 \\
& B_{1}=(1+R) B_{0}
\end{aligned}
$$

The stock price process however is a stochastic one. More specifically we assume that today's stock price $S_{0}$ is known, and at time $t_{1}$ the price will either go up, $u S_{0}$, with probability $p_{u}$ or down, $d S_{0}$, with probability $p_{d}=1-p_{u}$ where $0<d<u$ and $u, d, p_{u}, p_{d}$ are known variables. We also introduce the stochastic variable $Z$ :

$$
Z=\left\{\begin{array}{l}
u, \text { with probability } p_{u} \\
d, \text { with probability } p_{d}
\end{array}\right.
$$

and we can rewrite the stock price process as following:

$$
\left\{\begin{array}{l}
S_{0}=s \\
S_{1}=s \cdot Z
\end{array}\right.
$$

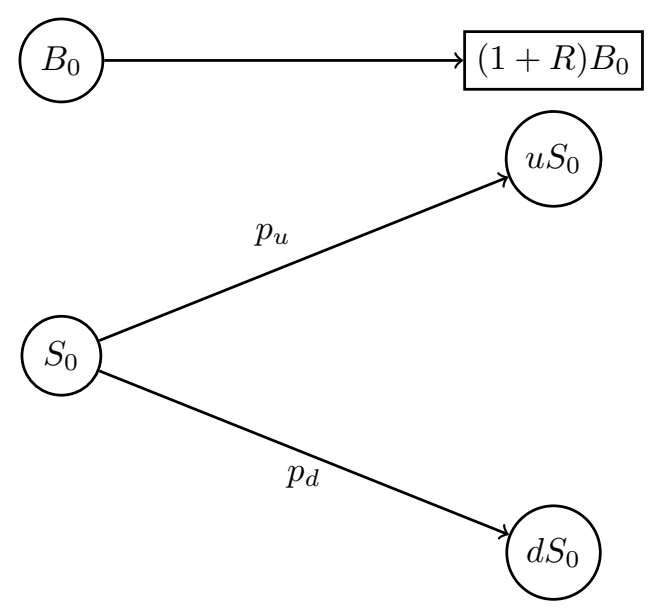

Figure 1: Price process in the one-period binomial model 
Definition 2.1. Let $p(x, y) \in \mathbb{R}^{2}$ be a 2-tuple representing a portfolio $p$, where $x$ is the number of bonds in the portfolio at time $t$ and $y$ is the number of units of stocks. Then the value process of the portfolio is:

$$
V_{t}^{p}=x B_{t}+y S_{t} \Leftrightarrow\left\{\begin{array}{l}
V_{0}^{p}=x+y S_{0} \\
V_{1}^{p}=x(1+R)+y S_{0} Z
\end{array}\right.
$$

Definition 2.2. A financial derivative, and in our case, a stock option is any stochastic variable, $X=\Phi(s)$. The function $\Phi$ is called the contract function and is in the case of a call option defined below:

$$
\Phi(s)= \begin{cases}s-K & \text { if } s \geq K \\ 0 & \text { if } s \leq K\end{cases}
$$

where $K$ is the strike price.

The price of stock options mainly depends on three elements, the time to expiration, the underlying stock price, and the volatility of the stock.

Definition 2.3. If the condition

$$
d<1+R<u
$$

is satisfied the model is arbitrage free.

This means that there is an imbalance between two or more markets and there exist an opportunity to make a risk free profit. An example of an arbitrage portfolio is a portfolio $p$ with the value process

$$
\begin{cases}V_{0}^{p} & =0 \\ P\left(V_{1}^{p}>0\right) & =1\end{cases}
$$

To understand this, assume that $(1+R)>u>d$, thus investing in the bond will always give higher return than an investment in the stock which gives us an arbitrage opportunity. So, if we short the stock and invest all the money in the bond, we are sure to make a profit of either $s(1+R)-s u$ or $s(1+R)-s d$ depending on the stochastic process of the stock's value.

Definition 2.4. The financial derivative $X$ is said to be reachable if there exists a portfolio $p$ such that:

$$
P\left(V_{1}^{p}=X\right)=1
$$

then we say that $p$ is a hedging/replicating portfolio.

This means that the assets in the replicating portfolio will have the same properties and, most importantly, the same cash flows as the asset/assets it is replicating. Replicating portfolios are a fundamental part of option pricing and financial mathematics as a whole. We will later in the thesis see how they are used to minimise the risks for the option writer. 
Proposition 2.1 (Pricing principle 1). If a financial derivative $X$ is reachable with a replicating portfolio $p$, then the only way to price an option at $t=0$ without creating any arbitrage opportunity is:

$$
\Pi(0 ; X)=V_{0}^{p}
$$

where $\Pi(t ; X)$ is the price of $X$ at time $t$.

Proof. If we let

$$
\Pi(0 ; X)>V_{0}^{p}
$$

then we can sell the option for $\Pi(0 ; X)$ at $t=0$ and invest the money in the replicating portfolio $p$. The remaining money $\left[\Pi(0 ; X)-V_{0}^{p}\right]$ we can save. At time $t=1$ the option holder will receive $V_{1}^{p}$ which in turn will be balanced out by our replicating portfolio and we thus have made a risk-free profit of $\left[\Pi(0 ; X)-V_{0}^{p}\right]$.

Similarly, we prove that $\Pi(0 ; X)<V_{0}^{p}$ also leads to an arbitrage opportunity.

Proposition 2.2. Given that the binomial model is free of arbitrage, the arbitrage free price of an option $X$ at time $t=0$ is given by:

$$
\Pi(0 ; X)=\frac{1}{1+R} E^{Q}\{X\}=\frac{1}{1+R} \cdot\left\{\Phi(s u) q_{u}+\Phi(s d) q_{d}\right\}
$$

$Q$ is called a martingale measure and is a mathematical construction with

$Q(Z=u)=q_{u} \& Q(Z=d)=q_{d}$ and $q_{u}, q_{d} \geq 0$. The probabilities, $q_{u}$ and $q_{d}$ are called the risk neutral probabilities and are specified so that:

$$
S_{0}=\frac{1}{1+R} E^{Q}\left\{S_{1}\right\}
$$

The martingale probabilities are given by:

$$
q_{u}=\frac{(1+R)-d}{u-d} \text { and } q_{d}=\frac{u-(1+R)}{u-d}
$$

$X$ can also be replicated using:

$$
\begin{aligned}
& x=\frac{1}{1+R} \cdot \frac{u \Phi(s d)-d \Phi(s u)}{u-d} \\
& y=\frac{1}{s} \cdot \frac{\Phi(s u)-\Phi(s d)}{u-d}
\end{aligned}
$$


Let us sum this up with an example:

Example 2.1. We are going to calculate the price of an European call option, $X$, with strike price $K=230 E U R$, stock value, $S_{0}=200 E U R$, risk-free rate, $R=0.02, u=\frac{7}{5}, d=\frac{5}{7}, p_{u}=0.55$ and $p_{d}=0.45$.

This gives us the following price process of the stock:

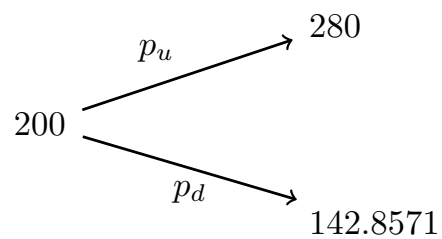

thus the contract function is defined as:

$$
\Phi\left(S_{1}\right)= \begin{cases}50 & \text { if } S_{1}>230 \\ 0 & \text { if } S_{1} \leq 230\end{cases}
$$

We can now use proposition 2.2 to calculate the martingale probabilities and the arbitrage free price:

$$
\begin{gathered}
\left\{\begin{array}{l}
q_{u}=\frac{35}{24}\left\{1.02-\frac{5}{7}\right\}=\frac{107}{240} \\
q_{d}=\frac{35}{24}\left\{\frac{7}{5}-1.02\right\}=\frac{133}{240}
\end{array}\right. \\
\Pi(0 ; X)=\frac{1}{1.02} E^{Q}\{X\}=\frac{1}{1.02}\left\{50 \cdot q_{u}+0 \cdot q_{d}\right\}=21+\frac{523}{612} \approx 21.8546
\end{gathered}
$$

and the claim is reachable with a replicating portfolio with the following composition using equation (14) in proposition 2.2:

$$
x=\frac{1}{1.02} \cdot \frac{-\frac{5}{7} \cdot 50}{\frac{24}{35}}=-51 \text { and } y=\frac{1}{200} \cdot \frac{50}{\frac{24}{35}}=\frac{35}{96},
$$

where we take a loan from the bank of 51 EUR and invest the money in $\frac{35}{96}$ shares of the stock. Thus the value of the portfolio at time $t=1$ is the following:

$$
\left\{\begin{array}{l}
V_{1}^{p}=-51 \cdot 1.02+\frac{35}{96} \cdot 200 \cdot \frac{7}{5}=50 \text { if } S_{1}=280 \\
V_{1}^{p}=-51 \cdot 1.02+\frac{35}{96} \cdot 200 \cdot \frac{5}{7}=0 \text { if } S_{1}=142.8571
\end{array}\right.
$$




\subsection{Multiperiod time model}

Before moving on we will briefly discuss the multiperiod model, again using Björk's definitions. The multiperiod model is just an extension of the one-period model, where $t$ denotes the step on the specific interval and $T$ is the expiration date given in years. The interval from 0 to $T$ is divided into $N$ equally long time-steps, $\Delta t=\frac{T}{N}$, where at each time-step the stock price can either go up or down just as before with probability $p_{u}$ and $p_{d}$ respectively. However, we will in this part of the thesis use Luenberger's definitions in Investment Science for $u$ and $d$ given below:

$$
\left\{\begin{array}{l}
u=e^{R \Delta t+\sigma \sqrt{\Delta t}} \\
d=e^{R \Delta t-\sigma \sqrt{\Delta t}}
\end{array}\right.
$$

where $\sigma$ is the volatility given in years.

The price process of the underlying assets for $t=0, \ldots T-\Delta t$ are the following:

$$
\begin{gathered}
\left\{\begin{array}{l}
B_{t+\Delta t}=e^{R \Delta t} B_{t}, \\
B_{0}=1
\end{array}\right. \\
\left\{\begin{array}{l}
S_{t+\Delta t}=S_{t} Z_{t}, \\
S_{0}=s
\end{array}\right.
\end{gathered}
$$

where all the $Z_{t}$ are I.I.D. and defined as above in the one period model.

Definition 2.5. A portfolio strategy is a stochastic process

$$
\left\{p_{t}\left(x_{t}, y_{t}\right): t=1, \ldots, T\right\}
$$

such that $p_{t}$ is a function of $S_{t-1}$. The value process corresponding to the portfolio $p$ is defined by:

$$
V_{t}^{p}=x_{t} e^{R \Delta t}+y_{t} S_{t}
$$

where $x_{t}$ is the amount invested in bonds and $y_{t}$ is the number shares of stocks invested at $t-1$ respectively and kept in the portfolio until time $t$.

Definition 2.6. A portfolio strategy $p$ is self-financing if the portfolio $p_{t}\left(x_{t}, y_{t}\right)$ equals the purchase value of the newly bought portfolio $p_{t+1}\left(x_{t+1}, y_{t+1}\right)$, i.e. if:

$$
x_{t} e^{R \Delta t}+y_{t} S_{t}=x_{t+1}+y_{t+1} S_{t}
$$


Definition 2.7. An arbitrage possibility is a self-financing portfolio $p$ with the properties:

$$
\begin{array}{r}
V_{0}^{p}=0, \\
P\left(V_{T}^{p} \geq 0\right)=1, \\
P\left(V_{T}^{p}>0\right)>0
\end{array}
$$

However as we can see that in our model with our newly defined $u$ and $d$ that they are separated by $e^{R \Delta t}$ which means that the model itself will not allow for any arbitrage opportunities and just as in the one-period model, we will use the $Q$ measurement, defined below:

Definition 2.8. The martingale measure is defined such that the following relationship holds:

$$
s=\frac{1}{e^{R \Delta t}} E^{Q}\left\{S_{t+1} \mid S_{t}=s\right\}
$$

Proposition 2.3 (Binomial algorithm). Consider a T-claim $X=\Phi\left(S_{T}\right)$. Then this claim can be replicated using a self-financing portfolio. If $V_{t}(k)$ denotes the value of the portfolio at node $(t, k)$, then $V_{t}(k)$ can be computed recursively by the scheme:

$$
\left\{\begin{array}{l}
V_{t}(k)=\frac{1}{e^{R \Delta t}}\left\{q_{u} V_{t+1}(k+1)+q_{d} V_{t+1}(k)\right\}, \\
V_{T}(k)=\Phi\left(s u^{k} d^{N-k}\right)
\end{array}\right.
$$

where $k$ denotes the number of up-moves that have been made and the martingale probabilities are given by:

$$
\left\{\begin{array}{l}
q_{u}=\frac{1}{e^{\sigma \sqrt{\Delta t}}+1} \\
q_{d}=\frac{1}{e^{-\sigma \sqrt{\Delta t}}+1}
\end{array}\right.
$$

The hedging portfolio is thus calculated by:

$$
\left\{\begin{array}{l}
x_{t}(k)=\frac{1}{e^{R \Delta t}} \frac{u V_{t}(k)-d V_{t}(k+1)}{u-d}, \\
y_{t}(k)=\frac{1}{S_{t-1}} * \frac{V_{t}(k+1)-V_{t}(k)}{u-d}
\end{array}\right.
$$

In particular, the arbitrage free price of the claim at $t=0$ is given by $V_{0}$. From the algorithm above it is also clear that we can obtain a risk neutral valuation formula.

Proposition 2.4. If a claim $X$ is reachable(i.e. there exists a self-financing portfolio $p$ such that $\left.P\left(V_{T}^{p}=X\right)=1\right)$ then the only arbitrage-free price process for $X$ is:

$$
\Pi(t ; X)=V_{t}^{p}, t=0, \ldots, T
$$

and the arbitrage free price at $t=0$ of the claim $X$ is given by:

$$
\Pi(0 ; X)=\frac{1}{\left(e^{R \Delta t}\right)^{N}} E^{Q}\{X\}=\frac{1}{\left(e^{R \Delta t}\right)^{N}} \sum_{k=0}^{N}\left(\begin{array}{l}
N \\
k
\end{array}\right) q_{u}^{k} q_{d}^{N-k} \Phi\left(s u^{k} d^{N-k}\right)
$$


We will in this example calculate the price process of a European call option in the multiperiod model and we will see that there are more than one way to get the option price at $t=0$.

Example 2.2. Let $s=300, \sigma=0.30, R=0.15, T=3$ months, $p_{u}=0.55$, $p_{d}=0.45, K=305$ we then have that $\Delta t=\frac{3}{12 \cdot 3}$ and

$$
\begin{gathered}
\left\{\begin{array}{l}
u=e^{0.15 \cdot \frac{1}{12}+0.30 \sqrt{\frac{1}{12}}} \\
d=e^{0.15 \cdot \frac{1}{12}-0.30 \sqrt{\frac{1}{12}}}
\end{array}\right. \\
\left\{\begin{array}{l}
q_{u}=\frac{1}{e^{0.30 \sqrt{\frac{1}{12}}}+1} \\
q_{d}=\frac{1}{e^{-0.30 \sqrt{\frac{1}{12}}}+1}
\end{array}\right.
\end{gathered}
$$

To find the price of the option at time $t=0$ we need to work backwards in the tree since the price of the option at $t=T$ is given by $\Pi(T ; X)=V_{T}^{p}$ and therefor deterministic. We can then look at the nodes on $t=\frac{2}{12}$ as starting prices for the stock in the one-period binomial model and use proposition 3.2 to get the option prices.
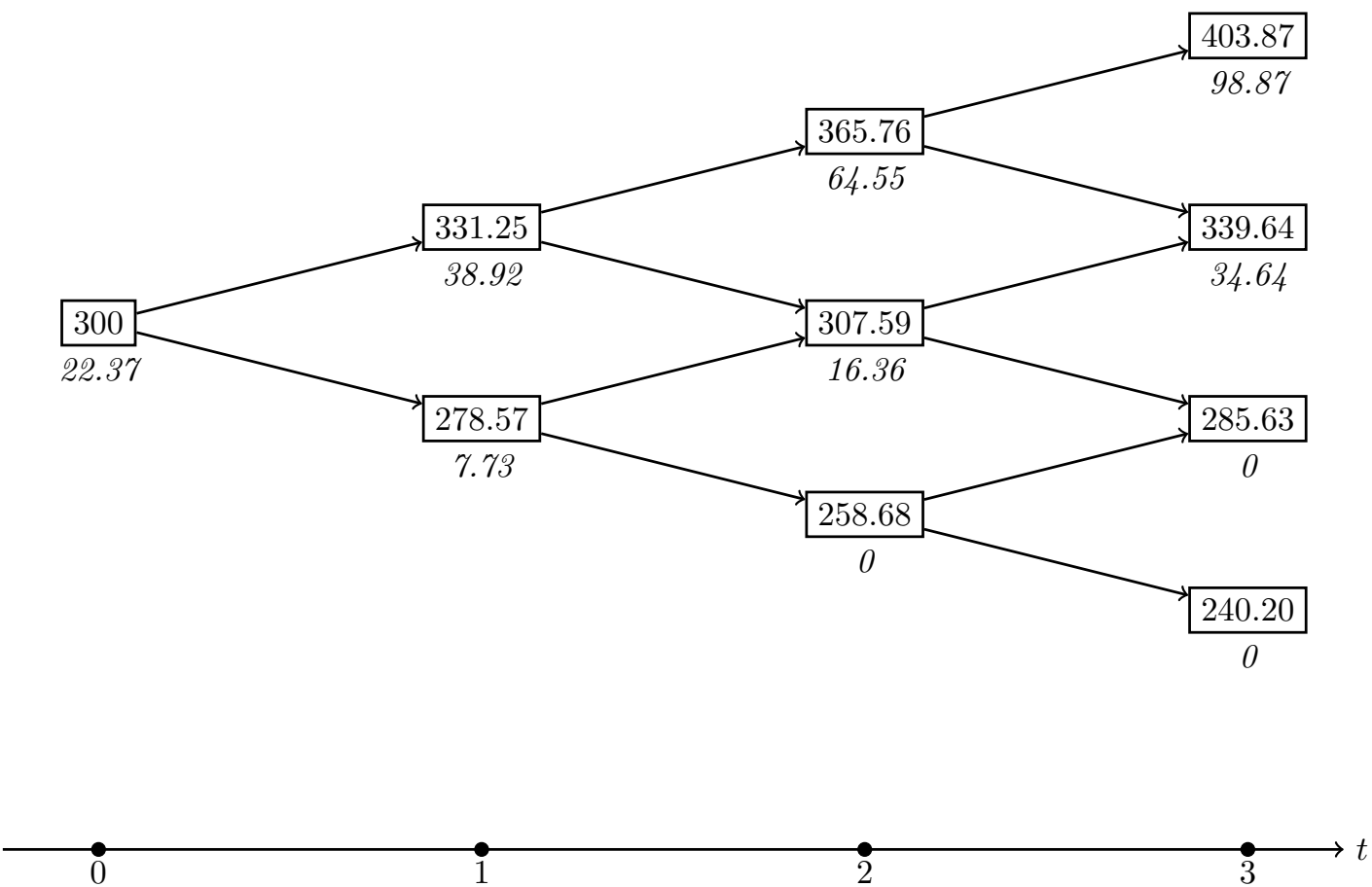

Figure 2: The price process of a option and its underlying assets given over 3 months 
Repeating the process for the nodes on $t=\frac{1}{12}$ we can calculate the option price at $t=0$ with the resulting tree graph given in Figure 2 where the numbers in the rectangles represent the stock price and the cursive numbers below the option price.

Using proposition 2.4 we get the same results:

$$
\begin{aligned}
\Pi(0 ; X) & =\frac{1}{\left(e^{0.15 / 12}\right)^{3}} \sum_{k=0}^{3} q_{u}^{k} q_{d}^{3-k} \Phi\left(s u^{k} d^{3-k}\right) \\
& =\frac{1}{\left(e^{0.15 / 12}\right)^{3}}\left(\left(\begin{array}{l}
3 \\
0
\end{array}\right) q_{d}^{3} \Phi\left(s d^{3}\right)+\left(\begin{array}{l}
3 \\
1
\end{array}\right) q_{u} q_{d}^{2} \Phi\left(s u d^{2}\right)+\left(\begin{array}{l}
3 \\
2
\end{array}\right) q_{u}^{2} q_{d} \Phi\left(s u^{2} d\right)+\left(\begin{array}{l}
3 \\
3
\end{array}\right) q_{u}^{3} \Phi\left(s u^{3}\right)\right) \\
& =0.9632(0+0+12.4046+10.8227) \approx 22.37
\end{aligned}
$$




\section{Volatility}

Volatility is an essential component in options pricing. According to Investopedia(see [3] and [4]), volatility is a statistical measure of the stock's fluctuation around the mean over a given period of time, given in percentage. Higher volatility is often associated with a higher risk for the option writer. If the volatility is high, the option premium will go up in price, and lower volatility will affect the premium conversely. Mathematically speaking, the generalised volatility for time $T$ is the standard deviation of the stock:

$$
\sigma=\sqrt{\operatorname{Var}\left\{\log \left(\frac{S_{T}}{S_{0}}\right)\right\} / T}
$$

\subsection{Exploring the choice of $\sigma$}

For a given volatility $\sigma$ over a time period $[0, T]$ the volatility will be constant in each step $t$, and because of the independence between the stock values the volatility over a given time period can be written as the sum of the variances

$$
\operatorname{Var}\left\{\log \left(\frac{S_{\Delta t}}{S_{0}}\right)+\ldots+\log \left(\frac{S_{T}}{S_{T-\Delta t}}\right)\right\}
$$

Thus one only have to look at the first step to examine $\sigma$.

$$
\sigma^{2}=E\left\{\log \left(\frac{S_{\Delta t}}{S_{0}}\right)^{2}\right\}-E\left\{\log \left(\frac{S_{\Delta t}}{S_{0}}\right)\right\}^{2}
$$

Since the stock price $S_{0}$ is deterministic we can set it to an arbitrary constant and let the $R=0$ for computational simplicity. If we let $S_{0}=1$ we get the following:

$$
\begin{aligned}
\sigma^{2} & =E\left\{\log \left(S_{1}\right)^{2}\right\}-E\left\{\log \left(S_{1}\right)\right\}^{2} \\
& =p \cdot \log (u s)^{2}+(1-p) \cdot \log (d s)^{2}-[p \cdot \log (u s)+(1-p) \cdot \log (d s)]^{2} \\
& =p\left[\log (u s)^{2}-\log (u s)^{2} p\right]+(1-p)\left[\log (d s)^{2}-\log (d s)^{2}(1-p)\right] \\
& -2 \log (u s) p \cdot \log (d s)(1-p) \\
& =\log (u s)^{2} p[1-p]+\log (d s)^{2}(1-p)[1-(1-p)]-2 \log (u s) p \cdot \log (d s)(1-p) \\
& =p(1-p)\left[\log (u s)^{2}+\log (d s)^{2}-2 \log (u s) \log (d s)\right]= \\
& =p(1-p)[\log (u s)-\log (d s)]^{2}=p(1-p)\left[\log \left(\frac{u s}{d s}\right)\right]^{2}= \\
& =p(1-p)\left[\log \left(\frac{u}{d}\right)\right]^{2} \Longrightarrow \sigma=\sqrt{p-p^{2}} \cdot \log \left(\frac{u}{d}\right) / \sqrt{\Delta t}
\end{aligned}
$$

Instead of $p$ we will use the martingale probabilities which can be obtained by solving

$$
u q+d(1-q)=e^{R \Delta t}
$$


since $R=0$ the expression will equal to one and we get that

$$
q=\frac{1-e^{-\sigma \sqrt{\Delta t}}}{e^{\sigma \sqrt{\Delta t}}-e^{-\sigma \sqrt{\Delta t}}}=\frac{1}{e^{\sigma \sqrt{\Delta t}}+1}
$$

substituting $\sigma$ with $A$ and plugging it into equation (30) we get:

$$
\begin{aligned}
\sigma & =\sqrt{\frac{1}{e^{A \sqrt{\Delta t}}+1}-\left(\frac{1}{e^{A \sqrt{\Delta t}}+1}\right)^{2}} \log \left(e^{2 A \sqrt{\Delta t}}\right) / \sqrt{\Delta t} \\
& =\sqrt{\frac{1}{e^{A \sqrt{\Delta t}}+1}-\left(\frac{1}{e^{A \sqrt{\Delta t}}+1}\right)^{2}} \cdot 2 A \\
\frac{\sigma^{2}}{4 A^{2}} & =\frac{1}{e^{A \sqrt{\Delta t}}+1}\left(1-\frac{1}{e^{A \sqrt{\Delta t}}+1}\right)
\end{aligned}
$$

Let $x=e^{A \sqrt{\Delta t}}+1$

$$
\begin{gathered}
\frac{\sigma^{2}}{4 A^{2}}=\frac{1}{x}\left(1-\frac{1}{x}\right)=\frac{x-1}{x^{2}} \\
x^{2}-\frac{4 A^{2}}{\sigma^{2}} x+\frac{4 A^{2}}{\sigma^{2}}=0 \\
\left(x-\frac{2}{\sigma} A\right)^{2} \Rightarrow x=\frac{2}{\sigma} A \\
e^{A \sqrt{\Delta t}}+1=\frac{2}{\sigma} A \\
\sigma=\frac{2}{e^{A \sqrt{\Delta t}}+1} A
\end{gathered}
$$

Thus when $\Delta t \rightarrow 0, \sigma$ tends to $A$ so the only way to price the option correctly is to let $A$ be as close to $\sigma$ as possible in this particular model so when $\Delta t$ goes to zero the two quantities will be arbitrary close. 


\section{Option prices and monotonicity in rate}

We will in this section explore the monotonicity of the option price in regard to the rate, $\mathrm{R}$, and what conditions needs to be put on $\Phi(s)$ to accomplish this. We will first look at this in the one-period model and later move on to the multiperiod model.

\subsection{Monotonicity in the one-period model}

We will start by exploring this for a call option, and than look at a more general case. For a call option, $\Phi(d s)$, will always equal zero, we can thus rewrite the equation and get:

$$
\begin{aligned}
\Pi(0 ; X)= & \frac{1}{1+R}\left\{\frac{(1+R)-d}{u-d} \Phi(u s)+\frac{u-(1+R)}{u-d} \Phi(d s)\right\}= \\
& \frac{1}{1+R}\left\{\frac{(1+R)-d}{u-d} \Phi(u s)\right\}
\end{aligned}
$$

taking the derivative of the function with respect to $R$ gives us the following expression,

$$
\begin{aligned}
\frac{\partial}{\partial R} \Pi(0 ; X)= & \frac{\Phi(u s)[(1+R)(u-d)]-(u-d)[\Phi(u s)(1+R-d)]}{(1+R)^{2}(u-d)^{2}}= \\
& \frac{\Phi(u s)(1+R)-\Phi(u s)(1+R-d)}{(1+R)^{2}(u-d)}=\frac{\Phi(u s) d}{(1+R)^{2}(u-d)}
\end{aligned}
$$

and since both $\Phi(u s), u$ and $d$ will greater than zero, and $d<u$ we have that $\Pi(0 ; X)$ will be a monotone non-decreasing function in $R \in \mathbb{R}$, without any additional conditions to $\Phi(u s)$.

Now looking at a more general case, we get:

$$
\begin{aligned}
\frac{\partial}{\partial R} \Pi(0 ; X)= & \frac{1}{u-d}\left\{\frac{1+R-R}{(1+R)^{2}} \Phi(u s)-\frac{1-d}{(1+R)^{2}} \Phi(u s)-\right. \\
& \left.\frac{1+R-R}{(1+R)^{2}} \Phi(d s)-\frac{u-1}{(1+R)^{2}} \Phi(d s)\right\}= \\
& \frac{\Phi(u s) d-\Phi(d s) u}{(1+R)^{2}(u-d)}
\end{aligned}
$$

The denominator will always be greater than zero because of $u>d$. So for $\Pi(0 ; X)$ to be a monotone increasing function of $R$ we need $\Phi(u s) d \geq \Phi(d s) u$. Rewriting the expression we get: 


$$
\begin{aligned}
\Phi(u s) d & \geq \Phi(d s) u \\
& \Leftrightarrow \\
\frac{\Phi(u s)}{u} & \geq \frac{\Phi(d s)}{d} \\
& \Leftrightarrow \\
\frac{\Phi(u s)}{u s} & \geq \frac{\Phi(d s)}{d s}
\end{aligned}
$$

Now, for a generalised variable $x \in \mathbb{R}_{>0}$, we know that if $\frac{\Phi(x)}{x}$ is a monotone increasing function this will imply that the condition above is satisfied. To see this, take for example an European call option, taking the derivative of the expression proves that, $\frac{\Phi(x)}{x}$, indeed is a monotone increasing function in $R$.

$$
\frac{\Phi(x)}{x}=\frac{x-K}{x} \Rightarrow \frac{d}{d x} \frac{\Phi(x)}{x}=\frac{K}{x^{2}} \text { for } \forall x \neq 0
$$

\subsection{Monotonicity in the multiperiod model}

Now looking the multiperiod model with $T=2$ and $N=2$,

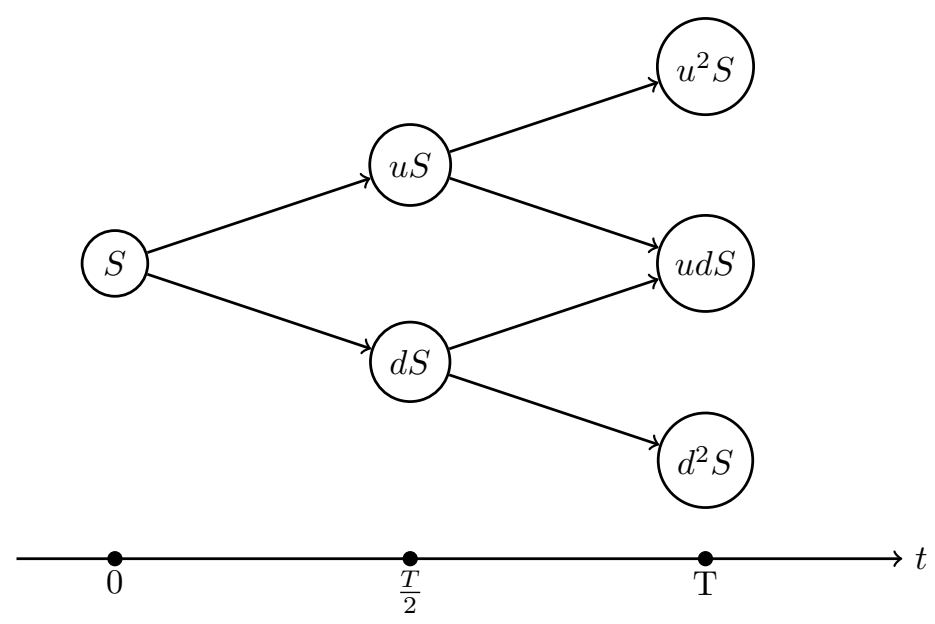

Figure 3: Price process in the two-period binomial model We get the following expressions for $u$ and $d$ :

$$
\left\{\begin{array}{l}
u=e^{r \frac{T}{2}+\sigma \sqrt{\frac{T}{2}}} \\
d=e^{r \frac{T}{2}-\sigma \sqrt{\frac{T}{2}}}
\end{array}\right.
$$


and we have $q$ such that:

$$
\begin{aligned}
& u q+d(1-q)=e^{r \frac{T}{2}}=\sqrt{u d} \\
& q=\frac{\sqrt{u d}-d}{u-d}=\frac{\sqrt{\frac{u}{d}}-1}{\frac{u}{d}-1}=\frac{e^{\sigma \sqrt{\frac{T}{2}}}-1}{e^{2 \sigma \sqrt{\frac{T}{2}}}-1}
\end{aligned}
$$

The option price at $t=0$ is given by:

$$
\Pi(0 ; X)=\frac{1}{e^{2 R}}\left(q^{2} \Phi\left(u^{2} s\right)+2 q(1-q) \Phi(u d s)+(1-q)^{2} \Phi\left(d^{2} s\right)\right)
$$

Differentiation with respect to $R$ gives:

$$
\begin{aligned}
\frac{\partial \Pi}{\partial R} & =2 \frac{1}{e^{2 R}} q^{2}\left(-\Phi\left(u^{2} s\right)+s u^{2} \Phi^{\prime}\left(u^{2} s\right)\right) \\
& +4 \frac{1}{e^{2 R}} q(1-q)\left(-\Phi(u d s)+s u d \Phi^{\prime}(u d s)\right)+2 \frac{1}{e^{2 R}}(1-q)^{2}\left(-\Phi\left(d^{2} s\right)+s d^{2} \Phi^{\prime}\left(d^{2} s\right)\right)
\end{aligned}
$$

Thus if

$$
x \Phi^{\prime}(x) \geq \Phi(x) \forall x \in \mathbb{R}
$$

then

$$
\frac{\partial \Pi}{\partial R} \geq 0
$$

and we have shown if equation (44) holds the option price will be monotone increasing with respect to the risk-free rate $R$, in the multiperiod binomial model for $N=2$. This can also readily be extended into N-periods as well and we can end this thesis by formulating a proposition:

Proposition 4.1. Consider an option $X$ in the N-period binomial model, then the option price will be monotone increasing in the risk-free rate $R$ if the following condition is satisfied:

$$
x \Phi^{\prime}(x) \geq \Phi(x) \forall x \in \mathbb{R}
$$




\section{References}

[1] Tomas Björk, Arbitrage Theory in Continuous Time, Oxford University Press; 2nd edition (May 6, 2004), Chapter 2.

[2] David G. Luenberger, Investment Science, Oxford University Press;(New York, 1998), Chapter 11-12.

[3] Justin Kuepper: Volatility, https://www.investopedia.com/terms/v/volatility.asp, (Apr 16, 2021)

[4] J.B. Maverick: How Does Implied Volatility Impact Options Pricing?, https://www. investopedia.com/terms/v/volatility.asp, (Jan 5, 2021) 